\title{
Low adherence to a prehospital continuous positive airway pressure treatment protocol following implementation in The Netherlands
}

\author{
Desiree Monique van der Lugt ${ }^{1 *}$, Anita W. Lekx ${ }^{1}$, Marieke M. G. J. Versteegen ${ }^{1}$, Dennis G. Barten ${ }^{1}$, \\ Loes Janssen ${ }^{2}$ and Oliver Thomas ${ }^{3,4}$
}

${ }^{1}$ Department of Emergency Medicine, VieCuri Medical Centre, Venlo, The Netherlands; ${ }^{2}$ Department of Clinical Epidemiology, VieCuri Medical Centre, Venlo, The Netherlands; ${ }^{3}$ Department of Intensive Care, VieCuri Medical Centre, Venlo, The Netherlands; ${ }^{4}$ Regional Ambulance Service LimburgNoord, Venlo, The Netherlands

\begin{abstract}
Background: In 2015, continuous positive airway pressure (CPAP) was incorporated in the treatment protocol of emergency medical services (EMS) in the Netherlands for acute respiratory failure. Potentially, CPAP has a positive effect on intubation rates, intensive care unit (ICU) admissions and mortality. Our aim was to study these effects. Secondary, we investigated the extent of CPAP use. Materials and Methods: This observational study compared two 6-month periods, before and after the introduction of prehospital CPAP (PCPAP). Patients were included if they were brought in by ambulance with a pulmonary cause of respiratory failure. Data were collected on blood gas results, Emergency Department length of stay, intubation, ICU admission, and mortality. In addition, a survey was held among EMS workers. Results: The before group contained 54 and the after group 46 patients. Only three patients (7\%) in the after group were treated with PCPAP, while all 46 patients fulfilled the inclusion criteria. According to the survey, reasons for not applying CPAP were: short distance to the hospital, too little knowledge about CPAP for respiratory failure with a suspected pulmonary cause, mask discomfort, and satisfying results on standard therapy alone. Conclusion: In this cohort, the use of PCPAP treatment was very low, although most patients (70\%) that fulfilled the inclusion criteria were admitted to the ICU. EMS workers did not feel familiar with the application of CPAP for patients with pulmonary dyspnea, despite training. Furthermore, mask discomfort showed to be an important factor, as well as the relatively short prehospital distances in the Netherlands. These results endorse the need for feasible prehospital protocols and the importance of patient comfort in the decision to provide PCPAP. More research is needed to evaluate if PCPAP in densely populated areas is beneficial.
\end{abstract}

Key words: Continuous positive airway pressure. Emergency department. Respiratory insufficiency.

\section{Correspondence:}

Desiree Monique van der Lugt

Department of Emergency Medicine

VieCuri Medical Centre

P.O. Box 1926, 5900 BX Venlo Date of reception: 11-10-2018

The Netherlands

Date of acceptance: 26-11-2018

E-mail: mvdlugt@ viecuri.nl

DOI: 10.24875/REIE.M19000003
Available online: 08-02-2019

Rev Educ Investig Emer. 2019;1(1):16-22 www.medicinadeemergencias.com

2604-6520 ( 2019 Sociedad Mexicana de Medicina de Emergencia, AC. Publicado por Permanyer México SA de CV. Este es un artículo Open Access bajo la licencia CC BY-NC-ND (http://creativecommons.org/licenses/by-nc-nd/4.0/). 


\section{Introduction}

Respiratory distress is a common reason for patients to visit the emergency department (ED). It often results from pulmonary conditions, such as chronic obstructive pulmonary disease (COPD) asthma and pneumonia ${ }^{1}$. Most patients with respiratory failure need to be admitted to an intensive care unit (ICU) for mechanical ventilation. Several studies have shown positive effects on mortality and length of hospital stay by starting early non-invasive ventilation on patients with respiratory failure in the $\mathrm{ED}^{2-4}$. However, little is known about the effect of using non-invasive ventilation or continuous positive airway pressure (CPAP) in the prehospital setting. A systematic review of 6 studies on prehospital CPAP (PCPAP) concluded that it has a positive effect on intubation rates and mortality in patients who are in acute respiratory failure ${ }^{5}$. The question is whether this would have the same outcome in the Netherlands where hospital density is high, and therefore the distance to the nearest hospital is short compared to other countries $^{6}$. However, more research is needed to further support this evidence?

CPAP is a form of non-invasive ventilation providing positive airway pressure during inhalation and expiration through a facial mask. It can be applied if standard care (supplemental oxygen, nebulizers, and hydrocortisone) is not sufficient to treat patients with respiratory failure $5,7,8$.

On April 1, 2015, CPAP was introduced in the prehospital treatment protocol in the Netherlands for patients with acute respiratory failure. In this protocol, the decision to apply CPAP is based on vital signs and the clinical decision by emergency medical services (EMS) personnel. The type of CPAP mask could be different between EMS regions.

In this study, we evaluated the introduction of PCPAP on the intubation rate, ICU admission rate, and mortality after the implementation of this technique in one of the 25 Dutch EMS regions (Northern Limburg). Furthermore, the extent of CPAP use was assessed. This is the first study in which PCPAP for patients with pulmonary dyspnea in the Netherlands is investigated.

\section{Materials and methods}

\section{Study design and setting}

This retrospective observational study was conducted in the ED of VieCuri Medical Center in Venlo, a teaching hospital in the southeastern part of the Netherlands with adherence of 280.000 patients. This hospital almost exclusively receives patients from the EMS region Northern Limburg, which is one of the 25 Dutch EMS regions. In this region, EMS workers were provided with Boussignac CPAP ( ${ }^{\circledR}$ Vygon) masks. All ED physicians, ED nurses, and EMS workers were given a 2 hours theoretical and practical training on CPAP use, followed by a brief refresher course a few months later. Based on the estimated amount of patients with respiratory failure visiting the ED, our goal was to include approximately 50 patients in both periods.

Two periods were compared; from October 1, 2014, to March 31, 2015 (before implementation of PCPAP) and October 1, 2015, to March 31, 2016 (after implementation). These months were chosen since patients with respiratory problems most often present in autumn and winter. In addition, we did not include the $1^{\text {st }}$ month directly after the introduction of CPAP to exclude the implementation period. Ethics approval was obtained from VieCuri Medical Center Research Ethics Committee.

\section{Inclusion and exclusion criteria}

Patients were included if they were brought in by ambulance and presented with dyspnea. Dyspnea was defined as a respiratory rate $>25 / \mathrm{min}$, pronounced use of accessory muscles, and/or an oxygen saturation $<95 \%$ (or $<88 \%$ in case of known COPD) without sufficient effect of conventional oxygen therapy or the use of a non-rebreathing mask. The same criteria are used to define insufficient respiration in the EMS guideline. The decision to apply CPAP, however, is also based on a clinical decision by EMS personnel.

Exclusion criteria were: age $<18$ years, no referral by ambulance, cardiac patients, non-ICU policy, prehospital intubation or pulmonary arrest, or dyspnea due to pneumothorax or pulmonary embolisms or suffocation. Patients with a contraindication for CPAP use were also excluded.

\section{Data collection}

Electronic patient records were used for screening and enrolment by the first author and data were collected on a case report form. When there was doubt about including a patient, the second reviewer was consulted. Ambulance medical forms are imbedded in the electronic patient record.

Age, sex, length of stay (ICU and hospital), use of PCPAP (second period), ICU admission, intubation, endotracheal intubation days, and 30-day mortality were recorded. Furthremore, first arterial blood gas results 
Table 1. Baseline characteristics

\begin{tabular}{|l|c|c|c|}
\hline & Group 1 & Group 2 & p-value \\
\hline Population (n) & 54 & 46 & - \\
\hline Age in years (SD) & $68(12)$ & $64(13)$ & 0.13 \\
\hline Male sex (\%) & $35(65)$ & $24(52)$ & 0.20 \\
\hline $\mathrm{pH}$ (SD) & $7.31(0.11)$ & $7.30(0.13)$ & 0.75 \\
\hline $\mathrm{pCO}_{2}^{*}$ (range) & $7.3(2.9-17.3)$ & $7.8(3.4-21.4)$ & 0.71 \\
\hline $\mathrm{pO}_{2}{ }^{*}$ (range) & $10(3.2-46.6)$ & $9.7(4.5-37.4)$ & 0.25 \\
\hline $\mathrm{CRP}^{* *}$ (range) & $43.5(1-468)$ & $62(1.6-484)$ & 0.38 \\
\hline Leukocytes count & $14.2(4.5-38.8)$ & 0.60 \\
\hline
\end{tabular}

Age and $\mathrm{pH}$ are presented as mean and standard deviation (SD); $\mathrm{pCO}_{2^{\prime}} \mathrm{pO}_{2^{\prime}}$ CRP and leukocytes count are presented as median and range. Group 1: before CPAP implementation, Group 2: after CPAP implementation. ${ }^{*} \mathrm{kPa} ;{ }^{* *} \mathrm{mg} / \mathrm{L} ;{ }^{* * *} 10^{*} \mathrm{E} 9 / \mathrm{L}$

were collected, as well as markers of inflammation (CRP [mg/L] and leukocytes).

\section{End points}

The primary outcomes were the intubation rate, number of ventilator days and length of ICU admission and secondary outcome was 30-day mortality.

In addition, a survey was held among EMS workers to identify reasons for not using PCPAP.

\section{Survey}

After collecting all data, we came to the conclusion that CPAP was often not applied on patients that fulfilled the inclusion criteria. Therefore, we conducted a survey among EMS workers about opinions on and personal experiences with PCPAP, as well as reasons for not using this technique. The survey consisted of four (2 open and 2 yes/no) questions and was spread among all EMS workers through their internal network web server.

\section{Statistical analysis}

Data analysis was performed in SPSS version 22 for Windows. Descriptive statistics were calculated, including means and standard deviations, or medians and ranges. Baseline characteristics and outcomes were compared between the two study groups. To identify possible predictors for ICU admission, we also compared baseline characteristics between patients admitted to the ICU and patients not admitted to the ICU.
For the categorical data, a Chi-square test or Fisher's exact test was performed to compare variables between the groups. For the continuous variables, we used an independent samples $t$-test when data were normally distributed. A Mann-Whitney U-test was used when data were not normally distributed. Missing data for the variables were not replaced.

\section{Results}

Between October 1, 2014, and March 31, 2015, 803 pulmonary patients visited the ED of which 54 patients (Group 1) fulfilled the inclusion criteria. Of these patients, $35(65 \%)$ were male. Between October 1, 2015, and March 31, 2016, 46 patients (Group 2) out of 630 patients met the inclusion criteria. Of these 46 patients, 24 (52\%) were male. The baseline characteristics of the two groups were comparable and are shown in table 1.

Patients presented with multiple diagnoses; COPD was noted most frequently, followed by pneumonia/ pulmonary sepsis, pulmonary edema, a combination of diagnoses, or dyspnea with an unknown cause.

\section{Outcome}

Between October 2015 and March 2016, 46 patients with a CPAP indication were identified. Only three of these patients $(6.5 \%)$ received PCPAP treatment in the ambulance. Another five patients (10.8\%) received CPAP in the ED. All three patients who received PCPAP continued this treatment in the ED (Table 2). The oxygen flow applied in the ambulance was higher in Group 2 than in Group 1, $12 \mathrm{~L} / \mathrm{min}$ and $6 \mathrm{~L} / \mathrm{min}$, 
Table 2. Main results

\begin{tabular}{|l|c|c|c|}
\hline & Group $\mathbf{1}(\mathbf{n}=\mathbf{5 4})$ & Group $\mathbf{~ ( n = ~ 4 6 ) ~}$ & p-value \\
\hline Patients receiving prehospital CPAP & Not available & 3 & - \\
\hline Patients receiving CPAP in ED & Not available & 8 & 0.09 \\
\hline Oxygen flow in ambulance in L/min (range) & $6(0-15)$ & $12(1-15)$ & 0.49 \\
\hline ICU admissions (\%) & $34(63)$ & $32(70)$ & 0.03 \\
\hline Median days on ICU (range) & $8(1-41)$ & $4.5(1-54)$ & 0.70 \\
\hline Median days in hospital (range) & $12(2-86)$ & $10.5(1-54)$ & 0.70 \\
\hline Non-invasive ventilation use ICU (\%) & $31(91)$ & $27(87)$ & 0.93 \\
\hline Intubation rates (\%) & $8(24)$ & $7(23)$ & 1.00 \\
\hline Days of mechanical ventilation (range) & $5.5(4-18)$ & $6.5(2-37)$ & 0.54 \\
\hline In-hospital mortality (\%) & $7(13)$ & $8(17)$ & 1.00 \\
\hline 30-day mortality (\%) & $3(6)$ & $3(7)$ & \\
\hline
\end{tabular}

Group 1: before CPAP implementation, Group 2: after CPAP implementation. $\mathrm{O}_{2}$ use, median days ICU/hospital and intubation days are presented as median and range. CPAP: continuous positive airway pressure; ICU: intensive care unit; ED: emergency department

respectively, although not significantly different. This variable was missing in 11 patients; 9 in the first group and 2 in the second group.

In the first group, there were $34 \mathrm{ICU}$ admissions $(63 \%)$, compared to $32(70 \%)$ in the second group $(p=0.49)$. The only significant difference between the two groups was the median ICU length of stay; 8 days in the first period and 4.5 days in the second period. As expected, non-invasive ventilation was applied in almost all patients admitted to the ICU, some before or after invasive ventilation.

The median length of hospital admission was 12 days in Group 1 and 10.5 days in Group 2, which was not significantly different. In-hospital mortality was $13 \%$ in Group 1 and 17\% in Group 2.

\section{CPAP versus non-CPAP}

The variables $\mathrm{pH}, \mathrm{pCO}_{2}, \mathrm{pO}_{2}, \mathrm{CRP}$, and leukocytes of patients who received PCPAP were compared with the mean or median values of all patients not receiving PCPAP. However, no firm conclusions can be drawn, since only three patients were treated with CPAP.

The mean $\mathrm{pH}$ of the patients treated with CPAP was lower than the mean $\mathrm{pH}$ of patients not treated with CPAP, as displayed in table 3. In addition, their median $\mathrm{pCO}_{2}$ was higher than the median $\mathrm{pCO}_{2}$ in the nonCPAP patients. In two of three CPAP patients, CRP was low, while the leukocyte numbers were relatively high in all three patients.

\section{Predictive factors for ICU admission}

The patients who were admitted to the ICU had a significantly lower $\mathrm{pH}$ and higher $\mathrm{pCO}_{2}$ compared with those not admitted to the ICU (Table 4). The $\mathrm{pO}_{2}$ was not significantly different; however, the prehospital oxygen flow was lower in patients admitted to the ICU.

\section{Survey}

After collecting the data, we held a survey among EMS workers to find out reasons for the low use of PCPAP. 23 of the 64 EMS workers (36\%) who received the questionnaire completed the survey. 14 of them $(61 \%)$ used CPAP in the ambulance before, irrespective of the cause of dyspnea. To the question why CPAP frequently was not applied in pulmonary patients who fulfilled the treatment criteria, the following reasons were given (in order of frequency): mask discomfort, short distances to the hospital, little knowledge about CPAP for COPD/pneumonia patients, and fear for $\mathrm{CO}_{2}$ accumulation and satisfying results on standard therapy alone. Although standard prehospital therapy was sufficient to transport the patient to the ED, admission and additional treatments were always necessary.

Among EMS workers, a majority $(61 \%)$ indicated that the mask was not user-friendly. Nevertheless, $90 \%$ of the responders believe that CPAP is of additional value in the prehospital setting, even in the Netherlands, where hospital density is relatively high. 
Table 3. First blood gas analyses: CPAP vs non-CPAP

\begin{tabular}{|c|c|c|c|c|}
\hline & CPAP 1 & CPAP 2 & CPAP 3 & Non-CPAP $(n=97)$ \\
\hline Age in years (SD) & 80 & 66 & 44 & $66(12)$ \\
\hline Male sex $(\%)$ & Male & Female & Male & $57(59)$ \\
\hline $\mathrm{pH}(\mathrm{SD})$ & 7.23 & 7.06 & 7.23 & $7.31(0.12)$ \\
\hline $\mathrm{pCO}_{2}^{*}$ (range) & 9.4 & 11.4 & 12.8 & $7.6(2.9-21.4)$ \\
\hline $\mathrm{pO}_{2}{ }^{*}$ (range) & 16.5 & 12.4 & 28.6 & $9.7(3.2-46.6)$ \\
\hline $\mathrm{CRP}^{* *}$ (range) & 8.7 & 5.0 & 79.0 & $61(1-484)$ \\
\hline Leukocytes ${ }^{* *}$ (range) & 17.5 & 12.4 & 19.3 & $14(4.5-41.3)$ \\
\hline
\end{tabular}

Table 4. Predictive factors ICU admission

\begin{tabular}{|l|c|c|c|}
\hline & ICU $+(\mathbf{n}=\mathbf{6 6})$ & ICU - (n=34) & p-value \\
\hline Age in years (SD) & $64(12)$ & $70(12)$ & 0.03 \\
\hline Male sex (\%) & $36(55)$ & $23(67)$ & 0.20 \\
\hline $\mathrm{pH}^{*}$ (SD) & $7.28(0.12)$ & $7.37(0.09)$ & 0.00 \\
\hline $\mathrm{pCO}_{2}{ }^{* *}$ (range) & $8.8(2.9-21.4)$ & $5.7(3.4-9.4)$ & 0.00 \\
\hline $\mathrm{pO}_{2}{ }^{* *}$ (range) & $9.3(3.2-46.6)$ & $10.8(5.5-37.4)$ & 0.03 \\
\hline Prehospital oxygen flow (L/min) range) & $6(1-15)$ & $15(3-15)$ & 0.01 \\
\hline
\end{tabular}

Predictive factors for ICU admission were identified comparing patients with (ICU +) and without (ICU -) ICU admission. ${ }^{*} \mathrm{KPa}$; ${ }^{* *} \mathrm{mg} / \mathrm{L} ;{ }^{* * *} 10^{*} \mathrm{Eg} / \mathrm{L}$

\section{Discussion}

Respiratory distress is a common reason for patients to visit the ED. There is often an indication for non-invasive ventilation and admission to the ICU. In this study, we evaluated the implementation of PCPAP in one EMS region in the Netherlands. We, therefore, investigated two groups of patients, before and after introducing PCPAP in the treatment protocol. Intubation rates, ICU admission rate, and mortality were compared. Unfortunately, there was insufficient data to make firm conclusions on the clinical effects of PCPAP. In contrast, the key finding of this study was that PCPAP was not applied in the majority of patients who were in respiratory distress. Only three of 46 patients $(7 \%)$ who fulfilled the definition of respiratory failure received PCPAP. These patients had a low ED blood gas $\mathrm{pH}$ and a high $\mathrm{pCO}_{2}$ compared to those not treated with CPAP. The prehospital application of CPAP is partly based on clinical decision making, so one might assume that these patients' respiratory failure is clinically more apparent than in non-CPAP patients.

In two of the three CPAP patients, the CRP was low, and the leukocyte count relatively high compared to the non-CPAP group. This suggests that these patients were more distressed, but not due to severe infection. However, the number of patients is too low to draw conclusions.

To evaluate the low use of PCPAP, a survey among EMS workers was conducted. According to this survey, the Boussignac mask was not user-friendly and comfortable for both patient and staff. In addition, there was little knowledge about the use of CPAP in patients with COPD and/or pneumonia, despite training. Furthermore, EMS workers ascertained that prehospital distances are sometimes too short to start with CPAP. The results endorse the importance of proper education when policymakers introduce new treatments or techniques. Furthermore, the feasibility and application of prehospital treatments are highly dependent on 
prehospital distances. Finally, mask (dis)comfort was shown to be an important factor to skip PCPAP, so feasibility is also determined by choice of CPAP mask type. The difficulty is that every person is different and has different facial features. On our EMS the Boussignac CPAP face mask system was used with two available sizes. These masks did not fit all patients properly, resulting in mask discomfort, which was another reason for the limited use of PCPAP. Another reason for mask discomfort can be due to incorrect fitting. These important factors need to be addressed in the future.

Only a few studies have published about the effect of PCPAP in COPD or pneumonia patients. Most research on PCPAP has been conducted on patients with acute cardiogenic pulmonary edema. In a study performed in Germany, where physician-supported EMS are deployed, the authors found that CPAP was effective for patients with COPD and as well as acute cardiogenic pulmonary edema ${ }^{9}$. Sahu et al. evaluated the use of CPAP (in prehospital setting) applied by basic life support (BLS) providers and found that CPAP can be safely used by BLS providers with appropriate training. They also used a Boussignac oxygen-driven CPAP, and their training consisted of a $4 \mathrm{~h}$ program ${ }^{10}$. The study by Knox et al. performed in the United States, with a study design comparable to our study, showed that the use of CPAP $(n=215)$ led to a decrease in intubation rates in patients with an acute respiratory disorder with an odds ratio of $0.38^{11}$. It was not clear whether Know et al. used a Boussignac system or a different system. Their training was, like ours, $2 \mathrm{~h}$. The training we gave to our EMS workers, therefore, does not seem to be a factor for low adherence to the protocol.

There are no articles published yet on the use of PCPAP in the Netherlands for patients with respiratory failure due to COPD or pneumonia. However, Spijker et al. examined the use of Boussignac CPAP in patients with acute cardiogenic pulmonary edema and came to the same conclusion as we did: a large proportion of their patients were not treated according to the EMS protocol $^{12}$. Possibly, the feasibility of PCPAP in the Netherlands is lower than in other countries, which might be explained by the relatively high hospital density compared to many other parts of Europe or the United States.

The majority of patients $(70 \%)$ who fulfilled the inclusion criteria were admitted to the ICU. These findings indicate that the definition of respiratory failure in the EMS protocol correctly identifies patients at risk. However, the protocol needs to be adjusted and starting PCPAP more standardized.
More research is needed to confirm this statement. Future studies should focus on a more detailed analysis on prehospital decision-making by EMS workers to apply CPAP or not. Furthermore, the correlation between prehospital distances, mask (dis)comfort and CPAP use should be further studied. Finally, large-scale, prospective studies are warranted to properly evaluate the effects of PCPAP on patients with a pulmonary cause of respiratory failure, both in densely and thinly populated areas.

The results of this study gave rise to the implementation of another, more comfortable and easy-to-use CPAP mask type (flow-safe II EZ, Mercury Medical), after which PCPAP use increased. In the period of October 1, 2016-March 31, 2017, there were 26 patients who received PCPAP, and in 3 patients CPAP was started in the ED. This increase is probably largely due to the change of CPAP mask type. However, it cannot be excluded if better training also played a role.

\section{Limitations}

This study has several limitations. The study population was relatively small, and only three patients received PCPAP despite instructions and training. The probable reasons for the low use are explained in the survey section above. It is a retrospective study and the first author was not blinded for the results. Nevertheless, the chance of selection bias is low, because respiratory failure was clearly defined.

Patients with a cardiac cause of respiratory failure (such as cardiac asthma or cardiogenic pulmonary edema) presenting to the (separately located) cardiac ED were not included due to logistic reasons. Patients who presented to the cardiac ED with a pulmonary cause of respiratory failure are, therefore, possibly missed. Furthermore, no data were collected from two other hospitals that were located in the same EMS region.

The diagnosis at admission or discharge was not retrieved in this research. Often a combined pulmonary and cardiac etiology was described. Nevertheless, prehospital diagnosis is less important when initiating CPAP, because it is indicated by vital signs and not the diagnosis itself.

Another limitation was the low participation of the online EMS workers survey, despite several remembrances.

\section{Conclusion}

Following the implementation of a PCPAP guideline in one EMS region in the Netherlands, only a few 
patients were treated with PCPAP for respiratory failure due to a pulmonary cause. Therefore, no conclusions could be made on the effect of PCPAP on morbidity and mortality. A survey among EMS workers showed that the low adherence to the protocol was determined by a shortage in knowledge, short prehospital distances, mask discomfort, fear for $\mathrm{CO}_{2}$ accumulation, and satisfying results on standard therapy alone. However, most patients that had an indication for CPAP were admitted to the ICU, which suggests that the definition of respiratory failure in the EMS guideline correctly identifies patients at risk. Unfortunately, the question remains whether PCPAP is useful in pulmonary dyspnea. Future prospective studies are warranted to evaluate if PCPAP is beneficial and cost-effective in patients with respiratory failure with a pulmonary cause. Finally, policymakers should acknowledge that the introduction of certain prehospital treatments might seem worthy but are not always feasible to patients and their health-care providers.

\section{Declaration of interest}

The authors report no conflicts of interest. The authors alone are responsible for the content and writing of the paper.

\section{Funding}

This research received no specific grant from any funding agency in the public, commercial or not-for-profit sectors.

\section{Acknowledgment}

We would like to thank the EMS workers AmbulanceZorg Limburg-Noord for their cooperation and contribution to this research.

\section{References}

1. Center for Statistics Netherlands (CBS)-2012 Emergency Department Contacts per Diagnosis; 2016. Available from: https://www.cbs.nl/nl-nl/ maatwerk/2016/26/seh-contacten-gebaseerd-op-het-dis-2012. [Last accessed on 2017 Sep 09].

2. Nava S, Hill N. Non-invasive ventilation in acute respiratory failure. Lancet. 2009;374:250-9.

3. Bolton R, Bleetman A. Non-invasive ventilation and continuous positive pressure ventilation in emergency departments: where are we now? Emerg Med J. 2008;25:190-4.

4. Allison MG, Winters ME. Noninvasive ventilation for the emergency physician. Emerg Med Clin North Am. 2016;34:51-62.

5. Goodacre S, Stevens JW, Pandor A, et al. Prehospital noninvasive ventilation for acute respiratory failure: systematic review, network meta-analysis, and individual patient data meta-analysis. Acad Emerg Med. 2014;21:960-70.

6. Kommer GJ, Gijsen R, Kooistra M, Deuning CM. Beschikbaarheid, Specialisatie en Bereikbaarheid van Spoedeisende Hulp in Nederland; Analyse Gevoelige Ziekenhuizen. Bilthoven: Rijksinstituut voor Volksgezondheid en Milieu (RIVM); 2015. Available from: https://www.volksge zondheidenzorg.info/onderwerp/acute-zorg/regionaal-internationaal/seh\#node-reistijd-minuten-naar-dichtstbijzijnde-ziekenhuis-met-seh-met-ambulance. [Last accessed on 2016 Nov 11].

7. Bakke SA, Botker MT, Riddervold IS, Kirkegaard H, Christensen EF. Continuous positive airway pressure and noninvasive ventilation in prehospital treatment of patients with acute respiratory failure: a systematic review of controlled studies. Scand J Trauma Resusc Emerg Med. 2014;22:69.

8. Nielsen VM, Madsen J, Aasen A, et al. Prehospital treatment with continuous positive airway pressure in patients with acute respiratory failure: a regional observational study. Scand J Trauma Resusc Emerg Med. 2016;24:121.

9. Luiz T, Kumpch M, Grüttner J, Madler C, Viergutz T. Prehospital CPAP therapy by emergency physicians in patients with acute respiratory failure due to acute cardiogenic pulmonary edema or acutely exacerbated COPD. In Vivo. 2016;30:133-9.

10. Sahu N, Matthews P, Groner K, Papas MA, Megargel R. Observational study on safety of prehospital BLS CPAP in dyspnea. Prehosp Disaster Med. 2017;32:610-4.

11. Knox N, Chinwe O, Themba N, Joseph F, Hormoz A. Relationship between intubation rate and continuous positive airway pressure therapy in the prehospital setting. World J Emerg Med. 2015;6:60-6.

12. Spijker EE, de Bont $M$, Bax M, Sandel M. Practical use, effects and complications of prehospital treatment of acute cardiogenic pulmonary edema using the boussignac CPAP system. Int J Emerg Med. 2013;6:8. 